\section{Systemic Sclerosis and Outcome in Intensive Care Unit: Do Not Close the Door, Open the Right Protocol}

To the Editor:

We read with great interest the paper by Pène, et al regarding the outcome of patients with systemic sclerosis (SSc) in the intensive care unit (ICU) ${ }^{1}$.

The authors performed a retrospective single-center study on 41 patients with SSc admitted to the ICU in a 6-year period and evaluated the short-term and longterm mortality rates. Most patients had pulmonary involvement at baseline: pulmonary fibrosis on computed tomography scan in $71.7 \%$ of cases and severe reduction of forced vital capacity and DLCO in $42.4 \%$ and $85.7 \%$ of cases, respectively. When considering causes of ICU admission, acute respiratory failure (ARF) was the main reason $(65.8 \%)$, with pulmonary infections accounting for $34.1 \%$ of cases, ARF without any definite diagnosis for $12.2 \%$ of cases, and drug-induced pneumonitis and pneumothorax, 1 case each. A noninvasive ventilation (NIV) trial was attempted in 13 patients $(31.7 \%)$ and was successful in 8 cases, whereas 5 patients required endotracheal intubation. A total of 13 patients were intubated (31.7\%), but only 2 of them survived the hospital stay. The requirement of mechanical ventilation (MV) was considered the worst prognostic factor in this group of patients.

Despite having few data available regarding the outcome of ARF in rare interstitial lung diseases (ILD) such as the one associated to SSc, poor prognosis after endotracheal intubation and MV has been described in more frequent ILD, particularly in acute exacerbation of idiopathic pulmonary fibrosis (IPF) $)^{2,3}$.

There are some key aspects to take into account for a proper clinical extrapolation of these results.

First, the reason for such finding still needs to be clarified; however, it could be related both to baseline lung impairment and the ARF etiology. As an example, treatable causes of ARF such as pneumonia and pulmonary embolism may have a better outcome compared to acute exacerbation of ILD, for which there is no definitive treatment evidence ${ }^{4}$.

Notably, in the authors' analysis the extent of baseline pulmonary involvement was not predictive of worse outcome (requirement of endotracheal intubation). Further, no statistically significant difference was found between patients who died in the hospital and survivors regarding pulmonary baseline involvement.

Second, given the restrospective design of the study and the small sample size, no further analysis can be made on the relationship between the etiology of ARF and the study outcomes. A consistent proportion of patients with ARF (5 out of 27) were labeled as "ARF without any definite diagnosis," perhaps hiding in this group patients with acute exacerbation of ILD. Moreover, with the exception of pulmonary infections that were well represented, other treatable causes of ARF were too rare to allow any speculation.

Third, given the poor prognosis associated with MV, in recent years great attention has been paid to the role of NIV in patients with ILD. Aliberti, et $a l$ in a recent paper evaluated 60 patients with ARF in diffuse ILD who underwent an NIV trial ${ }^{5}$. Underlying diagnoses were mainly IPF (47\% of cases), idiopathic nonspecific interstitial pneumonia (NSIP) in 27\% of cases, and NSIP associated to connective tissue diseases in $13 \%$ of cases. The most frequent causes of ARF were pneumonia ( $42 \%$ ) and acute exacerbation of fibrosis (39\%). Our study showed a significant improvement in oxygenation during NIV treatment in patients with pneumonia as the underlying etiology, but not in patients with acute exacerbation of fibrosis, suggesting that etiology of ARF plays a role in determining NIV efficacy. However, no statistically significant difference was found in regard to clinical failure (defined as in-hospital mortality, endotracheal intubation, and extracorporeal membrane oxygenation use) between patients with pneumonia and acute exacerbation.

Pène and coworkers also underlined the importance of the right setting and timing to perform an NIV trial, to avoid the risk of delayed intubation ${ }^{1}$.

An important message to convey is the need for protocols to choose the patients who might benefit most from NIV and the implementation in clinical practice of flowcharts to evaluate NIV response, like the ones developed for pneumonia ${ }^{6}$ (Figure 1). NIV could also be an alternative treatment for elderly or poor-performance patients considered poor candidates for intubation, and those with a "do not intubate" order.

NIV can be considered a valuable option to treat severe ARF in patients with ILD; however, location and timing (high-dependency unit with continuous monitoring), together with medical and nursing staff skills are crucial points to optimize patient comfort and ventilatory efficiency and avoid delayed intubation.

The majority of studies performed to date are retrospective, single-center, or based on the experience of a few centers and with a small sample size. We are in need of larger prospective studies that may cast new light on the role of NIV in ILD during an episode of ARF, in particular focusing on different underlying etiologies.

\section{DIAGNOSTIC INVESTIGATIONS}

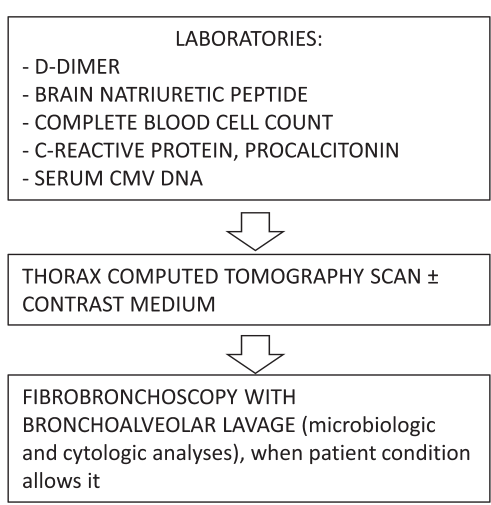

\section{ARF MANAGEMENT}

OPTIMIZE OXYGENATION \pm RESPIRATORY RATE
CONTROL
CONSIDER NIV TRIAL IF:
a) PaO2/FIO2 ratio $150-250$
b) $\mathrm{pH} 7.25-7.35$ and PaCO2 $>45 \mathrm{mmHg}$
c) Dyspnea/tachypnea (including accessory
muscle use and paradox abdominal
movement)
dBSENCE OF NIV CONTRAINDICATIONS:
neurological impairment, hemodynamic
instability, inability to protect the airways,
severe gastrointestinal bleeding,
multiorgan failure, undrained
pneumothorax, inability to fit the
interface.
PaO2/FiO2 ratio < 150 and/or pH $<7.25$
and a "do not intubate" order

Figure 1. Diagnostic and therapeutic investigations in acute respiratory failure in interstitial lung disease. CMV: cytomegalovirus; ARF: acute respiratory failure; NIV: noninvasive ventilation; $\mathrm{PaO} 2 / \mathrm{FiO} 2$ : ratio of partial pressure of arterial $\mathrm{O} 2$ to the fraction of inspired $\mathrm{O} 2 ; \mathrm{PaCO} 2$ : partial pressure of arterial $\mathrm{CO} 2$. 
PAOLA FAVERIO, MD, Department of Health Science, University of Milan Bicocca, Clinica Pneumologica; ALBERTO PESCI, MD,

Department of Health Science, University of Milan Bicocca, Clinica Pneumologica, Monza, Italy; ANTONIO M. ESQUINAS, MD, PhD, FCCP, Intensive Care and Non Invasive Ventilatory Unit, Hospital Morales Meseguer, Murcia, Spain. Address correspondence to Dr. P. Faverio, Department of Health Science, University of Milan Bicocca, Clinica Pneumologica, AO San Gerardo, Via Pergolesi 33, Monza, Italy.

E-mail: paola.faverio@gmail.com

\section{REFERENCES}

1. Pène F, Hissem T, Bérezné A, Allanore Y, Geri G, Charpentier J, et al. Outcome of patients with systemic sclerosis in the intensive care unit. J Rheumatol 2015;42:1406-12.

2. Gaudry S, Vincent F, Rabbat A, Nunes H, Crestani B, Naccache JM et al. Invasive mechanical ventilation in patients with fibrosing interstitial pneumonia. J Thorac Cardiovasc Surg 2014;147:47-53.

3. Vianello A, Arcaro G, Battistella L, Pipitone E, Vio S, Concas A, et al. Noninvasive ventilation in the event of acute respiratory failure in patients with idiopathic pulmonary fibrosis. J Crit Care 2014;29:562-7.

4. Collard HR, Moore BB, Flaherty KR, Brown KK, Kaner RJ, King TE Jr, et al; Idiopathic Pulmonary Fibrosis Clinical Research Network Investigators. Acute exacerbations of idiopathic pulmonary fibrosis. Am J Respir Crit Care Med 2007;176:636-43.

5. Aliberti S, Messinesi G, Gamberini S, Maggiolini S, Visca D, Galavotti V, et al. Non-invasive mechanical ventilation in patients with diffuse interstitial lung diseases. BMC Pulm Med 2014;14:194.

6. Ferrer M. Acute respiratory failure due to CAP. Eur Respir Monogr 2014;63:168-83.

J Rheumatol 2016;43:3; doi:10.3899/jrheum.151057 